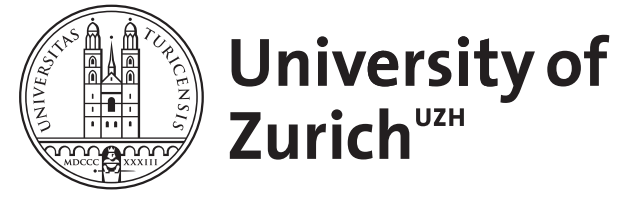

\title{
Statistical properties of the linear tidal shear
}

\author{
Desjacques, V ; Smith, R E
}

\begin{abstract}
Large-scale structures originate from coherent motions induced by inhomogeneities in the primeval gravitational potential. Here, we investigate the two-point statistics of the second derivative of the potential, the tidal shear, under the assumption of Gaussianity. We derive an exact closed form expression for the angular averaged, two-point distribution of the shear components which is valid for an arbitrary Lagrangian separation. This result is used to write down the two-point statistics of the shear eigenvalues in compact form. Next, we examine the large-scale asymptotics of the correlation of the shear eigenvalues and the alignment of the principal axes. The analytic results are in good agreement with measurements obtained from random realizations of the gravitational potential. Finally, we show that a number of two-point distributions of the shear eigenvalues are well approximated by Gaussian bivariates over a wide range of separation and smoothing scales. We speculate that the Gaussian approximation also holds for multiple point distributions of the shear eigenvalues. It is hoped that these results will be relevant for studies aimed at describing the properties of the (evolved) matter distribution in terms of the statistics of the primordial shear field
\end{abstract}

DOI: https://doi.org/10.1103/PhysRevD.78.023527

Posted at the Zurich Open Repository and Archive, University of Zurich

ZORA URL: https://doi.org/10.5167/uzh-13591

Journal Article

Accepted Version

Originally published at:

Desjacques, V; Smith, R E (2008). Statistical properties of the linear tidal shear. Physical Review D, 78(2):023527.

DOI: https://doi.org/10.1103/PhysRevD.78.023527 


\title{
Statistical properties of the linear tidal shear
}

\author{
Vincent Desjacques and Robert E. Smith \\ Institute for Theoretical Physics, University of Zürich, Winterthurerstrasse 190, CH-8057 Zürich, Switzerland \\ email: dvince@physik.uzh.ch, res@physik.uzh.ch
}

\begin{abstract}
Large-scale structures originate from coherent motions induced by inhomogeneities in the primeval gravitational potential. Here, we investigate the two-point statistics of the second derivative of the potential, the tidal shear, under the assumption of Gaussianity. We derive an exact closed form expression for the angular averaged, two-point distribution of the shear components which is valid for an arbitrary Lagrangian separation. This result is used to write down the two-point statistics of the shear eigenvalues in compact form. Next, we examine the large-scale asymptotics of the correlation of the shear eigenvalues, and the alignment of the principal axes. The analytic results are in good agreement with measurements obtained from random realizations of the gravitational potential. Finally, we show that a number of two-point distributions of the shear eigenvalues are well approximated by Gaussian bivariates over a wide range of separation and smoothing scales. We speculate that the Gaussian approximation also holds for multiple point distributions of the shear eigenvalues. It is hoped that these results will be relevant for studies aimed at describing the properties of the (evolved) matter distribution in terms of the statistics of the primordial shear field.
\end{abstract}

PACS numbers:

\section{INTRODUCTION}

In the currently favored $\Lambda \mathrm{CDM}$ cosmology, the galaxies and the large-scale structures we observe today are thought to arise from the hierarchical growth of initially tiny Gaussian fluctuations. Galaxy surveys [1] reveal that large-scale structures on scale $\sim 10-100 h^{-1} \mathrm{Mpc}$ form a predominantly filamentary network whose principal constituents - clusters, filaments and walls - accumulate on the boundary of large voids. Numerical simulations and semi-analytic approaches have been very successful in reproducing the observed matter distribution [2], whereas analytic models based on the spherical infall [3] predict mass functions, merging histories and spatial clustering of bound objects that are in reasonable agreement with the observations [4]. In parallel, several methods have been proposed to describe quantitavely the structures observed both in the Universe and in the simulations. The full hierarchy of correlation functions, the two-point statistics in particular [5], remains the most widely used statistical tools to distinguish between different scenarios of structure formation and constrain the cosmological parameters. Topological estimators such as Minkowski functionals [6] provide useful complementary information on the morphological characteristics of the filamentary network. Furthermore, various identification algorithms abstracting the spatial patterns in points, lines etc. have been proposed in an attempt to improve upon current topological measures [7].

Although the sequence in which large-scale structures form is still a matter of debate, many lines of evidence suggest that the filamentary pattern seen in observations and in N-body simulations is a consequence of the spatial coherence of the initial tidal shear [8]. While the spherical infall model [3] captures the essential features of gravitationally induced collapse, the primeval shear field has also been shown to play a crucial role in the formation of nonlinear structures [9]. As demonstrated in [10], the inclusion of non-sphericity in the collapse dynamics yields a better fit to the halo mass functions measured in N-body simulations. Yet another important manifestation of the tidal shear is the alignment of shape and angular momentum of objects $[11,12,13,14,15]$. Numerical studies of the $\Lambda \mathrm{CDM}$ cosmology report strong correlations in the alignment of galaxies, haloes, massive clusters or voids $[16,17,18,19,20]$, reflecting the coherence of the matter distribution out to large distances.

In the "Cosmic Web" picture outlined in [8], the correspondence between structures in the evolved density field and local properties of the linear tidal shear should, in principle, allow us to estimate the morphology of the matter distribution. In practice however, this correspondence has not been much exploited principally because of the lack of theoretical results. In spite of the progress made in the analysis of Gaussian random fields $[21,22,23]$ applied to the formation of large scale structures, the statistics of the shear has received little attention. Doroshkevich [24] first calculated the probability distribution of the shear eigenvalues and ascertained the amount of material being incorporated in sheet-like structures or pancakes. [25] reexamined the formation of these pancakes and derived a distribution function for the largest eigenvalue of the shear tensor. [26] computed conditional probability distributions for individual shear eigenvalues and obtained an analytic approximation to the halo mass function. Also, [27] explored the two-point correlation of the tidal shear components, but they did not discuss probability distributions.

In this paper, we carry out the analysis of the twopoint statistics of the linear tidal shear at two distinct (Lagrangian) positions. We extend the study of [28], who derived an expression for the shear 2-point statistics smoothed at two different scales, but evaluated at a 
single position. This work is essentially intended to provide theoretical results that could improve the statistical description of the tidal shear and, therefore, of the "Cosmic Web". Section $\S$ III is devoted to the calculation of the 2-point distributions. A careful examination of the small and large scale behavior of the 2-point distribution of shear components suggests a compact expression for the joint distribution of shear eigenvalues. This result is used in $\S \mathrm{IV}$ to explore the asymptotic behavior of the correlations of shear eigenvalues and principal axes. In $\S \mathrm{V}$, conditional 2-point distributions obtained from random realization of the linear shear field are compared with theoretical Gaussian distributions. It is argued that Gaussian multivariates provide a good description of the $n$-point distributions of shear eigenvalues at all separation and smoothing length. This suggests the possibility of implementing non-spherical collapse in current analytic models of structure formation using well-known results of the theory of Gaussian random fields.

\section{SHEAR}

The comoving Eulerian position of a particle can be generally expressed as a mapping

$$
\boldsymbol{x}=\boldsymbol{q}+\mathbf{S}(\boldsymbol{q}, a)
$$

where $\boldsymbol{q}$ is the Lagrangian (initial) position, $a$ is the scale factor and $\mathbf{S}$ is the displacement field. Continuity implies that the Eulerian density contrast $\delta(\boldsymbol{x}, a)$ is given by the reciprocal of the Jacobian for the transformation (1), $1+\delta(\boldsymbol{x}, a)=|\operatorname{det}(\partial \boldsymbol{q} / \partial \boldsymbol{x})|$. Singularities occur in this mapping whenever at least one of the eigenvalues is positive, signaling crossing of particle trajectories at that Eulerian point. The initial deformation tensor or strain field $\mathrm{D}_{i j}=\partial_{i} \mathbf{S}_{j}\left(\partial_{i} \equiv \partial / \partial q_{i}\right)$ thus encodes important information on the collapse of large-scale structures.

In the Zeldovich approximation, the displacement field is $\mathbf{S}(q, a)=-D_{+}(a) \nabla \Phi(\boldsymbol{q})$, where $\Phi(\boldsymbol{q})=$ $\phi(\boldsymbol{q}, a) / 4 \pi G \bar{\rho}_{m}(a) a^{2} D_{+}(a)$ is the perturbation potential, $\phi(\boldsymbol{q}, a)$ is the Newtonian gravitational potential, $\bar{\rho}_{m}$ is the average matter density and $D_{+}(a)$ is the linear growth factor (normalized such that $D_{+}(a)=1$ ) [5]. The strain tensor now is proportional to the second derivatives of the perturbation potential. For convenience, we will introduce the real, symmetric tensor

$$
\xi_{i j}(\boldsymbol{q})=\frac{1}{\sigma} \frac{\partial^{2} \Phi}{\partial q_{i} \partial q_{j}}(\boldsymbol{q}),
$$

where $\sigma$ is the rms variance of density fluctuations $\delta(\boldsymbol{q}) \equiv$ $\Delta \Phi(\boldsymbol{q})$ linearly extrapolated to the present time. We shall henceforth refer to $\xi_{i j}$ as the shear tensor. Notice that $\xi_{i j}$ is dimensionless while $\Phi(\boldsymbol{q})$ has the unit of (length) ${ }^{2}$. We will also assume that these fields are smoothed at some characteristic scale $R_{S}$ with a spherically symmetric window $W\left(r, R_{S}\right)$. Although many choices are possible for such a filtering function, we will adopt a top-hat filter throughout this paper, so that the variances are related to spherical volumes of radius $R_{S}$.

Let $\Lambda=\operatorname{diag}\left(\lambda_{1}, \lambda_{2}, \lambda_{3}\right)$ be the diagonalized shear tensor. For Gaussian initial conditions, the one-point probability distribution of the shear eigenvalues derived in [24] can be written as

$$
P\left(\lambda_{1}, \lambda_{2}, \lambda_{3}\right)=\frac{15^{3}}{8 \pi \sqrt{5}} e^{-Q_{1}(\Lambda, \Lambda)} \Delta(\lambda),
$$

where, for shorthand convenience,

$$
Q_{1}(\mathrm{X}, \mathrm{Y})=\frac{3}{4}[5 \operatorname{tr}(\mathrm{XY})-(\operatorname{tr} \mathrm{X})(\operatorname{tr} \mathrm{Y})]
$$

is some (indefinite) quadratic form over the space of real matrices, and

$$
\Delta(\lambda)=\operatorname{det}\left(\lambda_{i}^{3-j}\right)=\prod_{i<j}\left(\lambda_{i}-\lambda_{j}\right)
$$

is the Vandermonde determinant in the arguments $\lambda_{1}, \lambda_{2}, \lambda_{3}$. Our definition (2) of the shear tensor makes the probability $P\left(\lambda_{1}, \lambda_{2}, \lambda_{3}\right)$ independent of the filtering scale $R_{S}$. For instance, one finds that, for ambient field points, the probability of all three eigenvalues being positive is $P(+++)=0.08$, and that of the configurations $(++-)$ and $(+--)$ is 0.84 . Note, however, that these values depend strongly on the peak height, $\nu=\delta / \sigma$, of the region under consideration, the highest density peaks being predominantly spherical $[29,30]$.

\section{TWO-POINT STATISTICS}

Desjacques [28] extended the results of [24] to the joint statistics of the shear smoothed at different scales. However, he confined his calculation to the case in which the joint distributions are evaluated at a single Lagrangian position. Here we address the general case and calculate the joint distribution of the shear components $\xi_{i j}\left(\boldsymbol{q}_{1}\right)$ and $\xi_{k l}\left(\boldsymbol{q}_{2}\right)$ for arbitrary Lagrangian separations $\boldsymbol{r}=\boldsymbol{q}_{2}-\boldsymbol{q}_{1}$. We shall assume throughout this paper that the initial fluctuations are described by the Gaussian statistics. This assumption is remarkably well supported by the latest measurements of the Cosmic Microwave background (CMB) [31].

\section{A. Shear correlations}

We take the components $\xi_{i j}\left(\boldsymbol{q}_{1}\right)$ and $\xi_{k l}\left(\boldsymbol{q}_{2}\right)$ to be smoothed at two different (comoving) scales $R_{1}$ and $R_{2}$, respectively. The spectral parameter

$$
\gamma \equiv \frac{1}{\sigma_{1} \sigma_{2}} \int_{0}^{\infty} \operatorname{d} \ln k \Delta_{\delta}^{2}(k) \hat{W}\left(k, R_{1}\right) \hat{W}\left(k, R_{2}\right)
$$

$0 \leq \gamma \leq 1$, is a measure of the correlation between these scales. Here, $\Delta_{\delta}^{2}(k) \equiv k^{3} P_{\delta}(k) / 2 \pi^{2}$ is the dimensionless power spectrum of the density field, $\hat{W}\left(k, R_{i}\right)$ is the 
Fourier transform of $W\left(r, R_{i}\right)$, and $\sigma_{i}$ is the rms variance of density fluctuations $\delta(\boldsymbol{q})$ smoothed at scale $R_{i}$.

Statistical isotropy and symmetry imply that, in position space, the two-point correlation functions of an arbitrary symmetric tensor field $\xi_{i j}(\boldsymbol{q})$ must be of the form

$$
\begin{aligned}
& \left\langle\xi_{i j}\left(\boldsymbol{q}_{1}\right) \xi_{l m}\left(\boldsymbol{q}_{2}\right)\right\rangle=\Psi_{1}(r) \hat{r}_{i} \hat{r}_{j} \hat{r}_{l} \hat{r}_{m} \\
& \quad+\Psi_{2}(r)\left(\hat{r}_{i} \hat{r}_{l} \delta_{j m}+\hat{r}_{i} \hat{r}_{m} \delta_{j l}+\hat{r}_{j} \hat{r}_{l} \delta_{i m}+\hat{r}_{j} \hat{r}_{m} \delta_{i l}\right) \\
& \quad+\Psi_{3}(r)\left(\hat{r}_{i} \hat{r}_{j} \delta_{l m}+\hat{r}_{l} \hat{r}_{m} \delta_{i j}\right)+\Psi_{4}(r) \delta_{i j} \delta_{l m} \\
& \quad+\Psi_{5}(r)\left(\delta_{i l} \delta_{j m}+\delta_{i m} \delta_{j l}\right)
\end{aligned}
$$

where $r=\left|\boldsymbol{q}_{1}-\boldsymbol{q}_{1}\right|, \hat{r}_{i}=r_{i} / r$ is a unit vector and the functions $\Psi_{i}(r)$ depend on $r$ only. This is the most general ansatz for the isotropic sector of the fourth order correlation function $\left\langle\xi_{i j} \xi_{l m}\right\rangle(\boldsymbol{r})$. Symmetry requires that $\hat{r}$ appears in even number pairs. In the case of a scalar (spin-0) tensor such as the linear tidal shear defined in eq. (2), $\Psi_{2}=\Psi_{3}$ and $\Psi_{4}=\Psi_{5}$. Notice that equation (7) holds regardless of the statistical properties of the gravitational potential. However, when $\Phi(\boldsymbol{q})$ is Gaussian, the functions $\Psi_{i}$ may be conveniently expressed as

$$
\begin{aligned}
& \Psi_{1}(r)=\int_{0}^{\infty} \mathrm{d} \ln k \Delta^{2}(k) j_{4}(k r) \\
& \Psi_{3}(r)=\int_{0}^{\infty} \mathrm{d} \ln k \Delta^{2}(k)\left[-\frac{1}{7} j_{2}(k r)-\frac{1}{7} j_{4}(k r)\right] \\
& \Psi_{5}(r)=\int_{0}^{\infty} \mathrm{d} \ln k \Delta^{2}(k)\left[\frac{1}{15} j_{0}(k r)+\frac{2}{21} j_{2}(k r)+\frac{1}{35} j_{4}(k r)\right]
\end{aligned}
$$

where $\Delta^{2}(k) \equiv \Delta_{\delta}^{2}(k) \hat{W}_{1} \hat{W}_{2} /\left(\sigma_{1} \sigma_{2}\right)$ and $j_{\ell}(x)$ are spherical Bessel functions of the first kind. The $\Psi_{i}$ can be equivalently expressed in terms of the auxiliary functions $J_{n} \equiv n r^{-n} \int_{0}^{r} \mathrm{~d} s \psi(s) s^{n-1} \quad[27,32,33]$, where

$$
\psi(r)=\Psi_{1}(r)+10 \Psi_{3}(r)+15 \Psi_{5}(r)
$$

is the cross-correlation between the density enhancement $\delta / \sigma$ smoothed at two different scales. In the limit $r \rightarrow 0$, both $\Psi_{1}$ and $\Psi_{3}$ vanish while $\Psi_{5}$ tends towards $\gamma / 15$, so that $\psi \rightarrow \gamma$.

\section{B. Two-point probability distribution}

Owing to the symmetry of $\xi_{i j}$, only six components of the shear are independent. Following the notation of [23], let $\tilde{\xi}=\left\{\tilde{\xi}_{A}, A=1, \ldots, 6\right\}$ designate the six-dimensional vector whose components are equal to the components $i j=11,22,33,12,13,23$ of the shear tensor. The joint probability distribution $P\left(\xi_{1}, \xi_{2} ; \boldsymbol{r}\right)$ of the shear tensor $\xi_{1}=\xi_{i j}\left(\boldsymbol{q}_{1}\right)$ and $\xi_{2}=\xi_{i j}\left(\boldsymbol{q}_{2}\right)$ is given by a multivariate Gaussian whose covariance matrix $\mathrm{C}$ has 12 dimensions. This $12 \times 12$ matrix may be partitioned into four $6 \times$ 6 block matrices, $\mathrm{C}_{1}=\left\langle\tilde{\xi}_{1} \tilde{\xi}_{1}^{\top}\right\rangle$ in the top left corner, $\mathrm{C}_{2}=\left\langle\tilde{\xi}_{2} \tilde{\xi}_{2}^{\top}\right\rangle$ in the bottom right corner, $\mathrm{B}=\left\langle\tilde{\xi}_{1} \tilde{\xi}_{2}^{\top}\right\rangle$ and its transpose in the bottom left and top right corners, respectively, where

$$
\mathrm{C}_{1}=\mathrm{C}_{2}=\left(\begin{array}{cc}
\mathrm{M}_{1} / 15 & 0 \\
0 & \mathrm{I} / 15
\end{array}\right), \quad \mathrm{M}_{1}=\left(\begin{array}{lll}
3 & 1 & 1 \\
1 & 3 & 1 \\
1 & 1 & 3
\end{array}\right)
$$

and $\mathrm{I}$ is the $3 \times 3$ identity matrix.

Unlike $\mathrm{C}_{1}$ and $\mathrm{C}_{2}$, the cross-correlation matrix $\mathrm{B}$ generally is a function of the separation $r$. Using the harmonic decomposition of the tensor products $\hat{\boldsymbol{r}} \otimes \cdots \otimes \hat{\boldsymbol{r}}$ which appear in eq. $(7), \mathrm{B}(\boldsymbol{r})$ can be written as follows :

$$
\mathrm{B}(\boldsymbol{r})=\left(\begin{array}{ll}
\mathrm{B}_{1}(\boldsymbol{r}) & \mathrm{B}_{3}(\boldsymbol{r}) \\
\mathrm{B}_{3}(\boldsymbol{r}) & \mathrm{B}_{2}(\boldsymbol{r})
\end{array}\right)
$$

with $3 \times 3$ block matrices

$$
\begin{aligned}
& \mathrm{B}_{1}(\boldsymbol{r})=\frac{1}{15} \psi(r) \mathrm{M}_{1}+\sum_{\ell=2,4} \mathrm{~B}_{1}^{\ell, m}(r) Y_{\ell}^{m}(\hat{\boldsymbol{r}}) \\
& \mathrm{B}_{2}(\boldsymbol{r})=\frac{1}{15} \psi(r) \mathrm{I}+\sum_{\ell=2,4} \mathrm{~B}_{2}^{\ell, m}(r) Y_{\ell}^{m}(\hat{\boldsymbol{r}}) \\
& \mathrm{B}_{3}(\boldsymbol{r})=\sum_{\ell=2,4} \mathrm{~B}_{3}^{\ell, m}(r) Y_{\ell}^{m}(\hat{\boldsymbol{r}}) .
\end{aligned}
$$

$Y_{\ell}^{m}(\hat{\boldsymbol{r}})$ are spherical harmonics and $\mathrm{B}_{i}^{\ell, m}(r)$ are $3 \times 3$ matrices which satisfy $\left(\mathrm{B}_{i}^{\ell, m}\right)^{\dagger}=(-1)^{m} \mathrm{~B}_{i}^{\ell, m}\left(\mathrm{~B}_{i}(\boldsymbol{r})\right.$ are realvalued matrix). An explicit calculation of these matrices is not necessary as we will focus on the contribution of the monopole terms. Again, symmetry implies that only the harmonics with multipoles $\ell=0,2,4$ and even $m$ appear in the decomposition. Furthermore, it is worthwhile noticing that the joint probability density $P\left(\xi_{1}, \xi_{2} ; \boldsymbol{r}\right)$ is invariant under any arbitrary rotation of the coordinate system [34],

$$
P\left(\xi_{1}^{\prime}, \xi_{2}^{\prime} ; \boldsymbol{r}^{\prime}\right)=P\left(\mathrm{R} \xi_{1} \mathrm{R}^{\top}, \mathrm{R} \xi_{2} \mathrm{R}^{\top}, \mathrm{R}^{\top} \boldsymbol{r}\right)=P\left(\xi_{1}, \xi_{2}, \boldsymbol{r}\right)
$$

where primes denote quantities in the new coordinate frame. However, in a given coordinate system, its functional form changes when $\hat{\boldsymbol{r}}$ moves over the unit sphere. Namely, the transverse and longitudinal components of the two-point shear correlation vary with the orientation of the separation vector. Therefore, the anisotropic structure of $\left\langle\xi_{i j} \xi_{l m}\right\rangle(\boldsymbol{r})$ should come as no surprise [27].

\section{Angle average distribution}

We are primarily interested in the angular average of the two-point probability distribution of the linear tidal shear,

$$
P\left(\xi_{1}, \xi_{2} ; r\right)=\frac{1}{4 \pi} \int \mathrm{d} \Omega_{\hat{r}} P\left(\xi_{1}, \xi_{2} ; \boldsymbol{r}\right),
$$

which is a function of the separation $r$ solely. To get insight on the functional form of $P\left(\xi_{1}, \xi_{2} ; r\right)$, we will examine the small-scale $(r \ll 1)$ and large-distance asymptotic 
$(r \gg 1)$ behavior of the probability density $P\left(\xi_{1}, \xi_{2}, \boldsymbol{r}\right)$. In both regimes, the cross-correlation matrix $\mathrm{B}$ can be expressed as $\mathrm{B}(\boldsymbol{r})=\overline{\mathrm{B}}+\delta \mathrm{B}(\boldsymbol{r})$, where $\delta \mathrm{B}$ is a small perturbation and $\overline{\mathrm{B}}$ is the zero order contribution, which is either $\overline{\mathrm{B}}=\gamma \mathrm{C}_{1}$ (when $r \rightarrow 0$ ) or $\overline{\mathrm{B}}=0$ (when $r \rightarrow \infty$ ). The quadratic form which appears in the probability distribution $P\left(\xi_{1}, \xi_{2} ; \boldsymbol{r}\right)$,

$$
P\left(\xi_{1}, \xi_{2} ; \boldsymbol{r}\right)=\frac{1}{(2 \pi)^{6}|\operatorname{det} C|^{1 / 2}} e^{-Q\left(\xi_{1}, \xi_{2} ; r\right)},
$$

can be computed easily using Schur's identities. Here, $\operatorname{det} \mathrm{C}$ is the determinant of the covariance matrix C. Expanding the exponential in the perturbation $\delta \mathrm{B}$ and averaging over directions $\hat{r}$, we find

$$
\begin{aligned}
& \frac{1}{4 \pi} \int \mathrm{d} \Omega_{\hat{r}} \exp \left[-Q\left(\xi_{1}, \xi_{2} ; \boldsymbol{r}\right)\right] \\
& \quad \approx\left\{1-2 \frac{(\psi-\gamma)}{1-\gamma^{2}}\left[\frac{\gamma}{1-\gamma^{2}}\left(Q_{1}\left(\xi_{1}, \xi_{1}\right)+Q_{1}\left(\xi_{2}, \xi_{2}\right)\right)\right.\right. \\
& \left.\left.\quad-Q_{1}\left(\xi_{1}, \xi_{2}\right)\right]\right\} e^{-\left[Q_{1}\left(\xi_{1}, \xi_{1}\right)+Q_{1}\left(\xi_{2}, \xi_{2}\right)-2 \gamma Q_{1}\left(\xi_{1}, \xi_{2}\right)\right] /\left(1-\gamma^{2}\right)} \\
& \quad(r \ll 1), \quad(16) \\
& \approx\left[1+2 \psi Q_{1}\left(\xi_{1}, \xi_{2}\right)\right] e^{-Q_{1}\left(\xi_{1}, \xi_{1}\right)-Q_{1}\left(\xi_{2}, \xi_{2}\right)} \\
& \quad(r \gg 1), \quad(17)
\end{aligned}
$$

to first order in $\delta \mathrm{B}$. Interestingly, these perturbative expansions precisely match the small-scale and asymptotic large $r$ behavior of the function $\exp \left[-Q_{2}\left(\xi_{1}, \xi_{2}, r\right)\right]$, where

$$
Q_{2}\left(\xi_{1}, \xi_{2} ; r\right)=\frac{Q_{1}\left(\xi_{1}, \xi_{1}\right)+Q_{1}\left(\xi_{2}, \xi_{2}\right)-2 \psi Q_{1}\left(\xi_{1}, \xi_{2}\right)}{1-\psi^{2}} .
$$

This strongly suggests that the two-point probability distribution $P\left(\xi_{1}, \xi_{2} ; r\right)$ of the shear tensor may be written exactly as

$$
P\left(\xi_{1}, \xi_{2} ; r\right)=\frac{1}{20}\left(\frac{15}{2 \pi}\right)^{6}\left(1-\psi^{2}\right)^{-3} e^{-Q_{2}\left(\xi_{1}, \xi_{2} ; r\right)} .
$$

This distribution depends on the separation $r$ through the density correlation $\psi(r)$ only. Gaussianity and invariance under rotation requires that $P$ be a function of $\operatorname{tr}\left(\xi_{i}^{2}\right),\left(\operatorname{tr} \xi_{i}\right)^{2}, \operatorname{tr}\left(\xi_{1} \xi_{2}\right)$ and $\operatorname{tr} \xi_{1} \operatorname{tr} \xi_{2}$ solely. Although we have not been able to rigorously prove that equation (19) correctly describes the two-point distribution in the intermediate region, we have found that it agrees with the result of a direct numerical integration of eq. (14) (for various choices of $\xi_{1}, \xi_{2}$ and $r$ ) up to the numerical accuracy. Furthermore, the fact that, in the limit $r \ll 1$, eq. (19) reduces to the joint probability density derived in [28] is another indication of correctness.

\section{Joint distribution of the eigenvalues}

We now choose a coordinate system such that the coordinate axes are aligned with the principal axes of

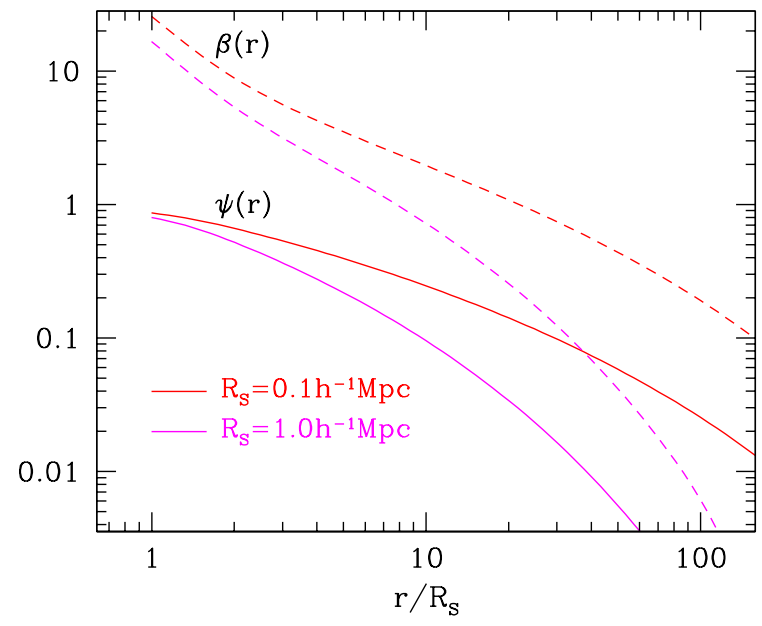

FIG. 1: The density correlation $\psi(r)$ and the parameter $\beta(r)$ (cf. text) as a function of the ratio $r / R_{S}$ for two different smoothing lengths $R_{S}=0.1$ and $1 h^{-1} \mathrm{Mpc}$.

$\xi_{1}$. Let $\Lambda_{1}$ and $\Lambda_{2}$ be the diagonal matrices consisting of the three ordered eigenvalues $x_{1} \geq x_{2} \geq x_{3}$ and $y_{1} \geq y_{2} \geq y_{3}$ of the deformation tensors $\xi_{1}$ and $\xi_{2}$, respectively. The principal axis are now labeled according to this ordering. With this choice of coordinate, $\xi_{1}=\Lambda_{1}$ and $\xi_{2}=\mathrm{R} \Lambda_{2} \mathrm{R}^{\top}$, where $\mathrm{R}$ is an orthogonal matrix that defines the orientation of the eigenvectors of $\xi_{2}$ relative to those of $\xi_{1}$. To preserve the orientation of the principal axis frames, we further impose the condition that the determinant of $\mathrm{R}$ must be +1 . Namely, $\mathrm{R}$ belongs to the special orthogonal group $\mathrm{SO}(3)$. The properties of the trace imply that $Q_{1}\left(\xi_{2}, \xi_{2}\right)=Q_{1}\left(\Lambda_{2}, \Lambda_{2}\right)$, while the term $Q_{1}\left(\xi_{1}, \xi_{2}\right)=Q_{1}\left(\Lambda_{1}, \mathrm{R} \Lambda_{2} \mathrm{R}^{\top}\right)$ depends on the rotation matrix.

To obtain the joint probability distribution of the ordered eigenvalues of the shear tensor, 'angular' variables, such as those appearing in $Q_{1}\left(\xi_{1}, \xi_{2}\right)$, have to be integrated over. The volume measure $\mathrm{d} \xi$ for the space of real $3 \times 3$ symmetric matrices can be expressed in terms of the non-increasing sequence of eigenvalues $\lambda_{i}\left(=x_{i}\right.$ or $\left.y_{i}\right)$ as

$$
\mathrm{d} \xi=8 \pi^{2} \Delta(\lambda) \mathrm{d}^{3} \lambda \mathrm{dR} .
$$

Here, $\mathrm{dR}$ is the Haar measure on the group $\mathrm{SO}(3)$ normalized to $\int \mathrm{dR}=1, \mathrm{~d}^{3} \lambda=\mathrm{d} \lambda_{1} \mathrm{~d} \lambda_{2} \mathrm{~d} \lambda_{3}$ and $\Delta(\lambda)$ is the Vandermonde determinant eq. (5). When the rotation matrices $\mathrm{R}$ is parametrised in terms of the Euler angles $0 \leq \varphi, \psi \leq 2 \pi, 0 \leq \vartheta \leq \pi$, the Haar measure takes the familiar form

$$
\mathrm{dR}=\frac{1}{8 \pi^{2}} \sin \vartheta \mathrm{d} \varphi \mathrm{d} \vartheta \mathrm{d} \psi
$$

Since the quadratic form $Q$ depends only on the relative orientation of the eigenvector triads of $\xi_{1}$ and $\xi_{2}$, we can immediately integrate over one of the $\mathrm{SO}(3)$ manifolds. The relevant volume is $8 \pi^{2} / 4=2 \pi^{2}$. The factor 4 comes 
from not caring whether the rotated axis points in the positive or negative direction [23]. The essential problem is the calculation of the integral over the manifold that defines relative, distinct triad orientations,

$$
\mathcal{I}=\int_{\mathrm{SO}(3)} \mathrm{dR} \exp \left[\beta \operatorname{tr}\left(\mathrm{R} \Lambda_{2} \mathrm{R}^{\top} \Lambda_{1}\right)\right]
$$

where we have defined $\beta(\psi)=(15 / 2) \psi /\left(1-\psi^{2}\right)$.

There is no analytic, closed-form solution to this integral, although an exact determinantal expression was derived when averaging over the unitary group $\mathrm{U}(\mathrm{N})[35$, 36]. An asymptotic representation can be obtained when $\beta \gg 1$. For reasonable values of $R_{S}$, this occurs when the separation $r$ is less than a few smoothing radii (see Fig. 1). In general, the integral (22) can be expressed as a hypergeometric series with the symmetric $3 \times 3$ matrices $\beta \Lambda_{1}$ and $\Lambda_{2}$ as argument (see Appendix $\S \mathrm{A}$ ). In the notation of [37], $\mathcal{I} \equiv{ }_{0} F_{0}^{(3)}$ where, at second order in $\beta$,

$$
\begin{array}{r}
{ }_{0} F_{0}^{(3)}\left(\beta \Lambda_{1}, \Lambda_{2}\right)=1+\frac{\beta}{3} \operatorname{tr} \Lambda_{1} \operatorname{tr} \Lambda_{2}+\frac{\beta^{2}}{18}\left(\operatorname{tr} \Lambda_{1}\right)^{2}\left(\operatorname{tr} \Lambda_{2}\right)^{2} \\
+\frac{\beta^{2}}{90}\left[3 \operatorname{tr}\left(\Lambda_{1}^{2}\right)-\left(\operatorname{tr} \Lambda_{1}\right)^{2}\right]\left[3 \operatorname{tr}\left(\Lambda_{2}^{2}\right)-\left(\operatorname{tr} \Lambda_{2}\right)^{2}\right] .(23)
\end{array}
$$

Higher order terms are intentionally omitted as they are not used in the present analysis.

A straightforward calculation shows that the eigenvalue joint probability distribution $P(x, y ; r)$ evaluates to

$$
\begin{aligned}
P(x, y ; r)= & \frac{15^{6}}{320 \pi^{2}}\left(1-\psi^{2}\right)^{-3}{ }_{0} F_{0}^{(3)}\left(\beta \Lambda_{1}, \Lambda_{2}\right) \\
& \times e^{-Q_{12}\left(\Lambda_{1}, \Lambda_{2} ; r\right)} \Delta(x) \Delta(y)
\end{aligned}
$$

where

$$
Q_{12}=\frac{Q_{1}\left(\Lambda_{1}, \Lambda_{1}\right)+Q_{1}\left(\Lambda_{2}, \Lambda_{2}\right)+\frac{3}{2} \psi\left(\operatorname{tr} \Lambda_{1}\right)\left(\operatorname{tr} \Lambda_{2}\right)}{\left(1-\psi^{2}\right)} .
$$

Notice that, in the limit $|\psi| \ll 1$, the joint probability distribution $P(x, y ; r)$ tends, as it should be, toward the product of the individual one-point probability distribution. Using Bayes' theorem, we easily derive a conditional distribution $P(x \mid y ; r)$ for the shear eigenvalues $x_{i}$ given the $y_{i} \mathrm{~s}$ and a separation $r$,

$P(x \mid y ; r)=\frac{15^{3}}{8 \pi \sqrt{5}}\left(1-\psi^{2}\right)^{-3}{ }_{0} F_{0}^{(3)}\left(\beta \Lambda_{1}, \Lambda_{2}\right) e^{-Q_{1 \mid 2}} \Delta(x)$,

where the quadratic form $Q_{1 \mid 2}\left(\Lambda_{1}, \Lambda_{2} ; r\right)$ is

$$
Q_{1 \mid 2}=\frac{Q_{1}\left(\Lambda_{1}, \Lambda_{1}\right)+\psi^{2} Q_{1}\left(\Lambda_{2}, \Lambda_{2}\right)+\frac{3}{2} \psi\left(\operatorname{tr} \Lambda_{1}\right)\left(\operatorname{tr} \Lambda_{2}\right)}{1-\psi^{2}} .
$$

A direct numerical integration convinced us that the probability distribution (26) is normalized to unity (we used the multi-dimensional integrator DCUHRE described in [39]). We believe that eqs (24) and (26) are exact expressions for the two-point and conditional probability distribution function of the shear eigenvalues. They generalize the results obtained in $[24,28]$.

\section{ASYMPTOTICS OF CORRELATION FUNCTIONS}

Instead of attempting a brute force calculation of the correlation functions through a direct integration of the probability density (24), we will examine the large scale asymptotic behavior solely. We will nonetheless show infer analytic approximations to the correlations of shear eigenvalues which are accurate on all scales.

\section{A. Eigenvalues}

In order to derive the correlation function for the eigenvalues of the shear tensor in the asymptotic regime $r \gg 1$ for which $\psi \ll 1$ and $\beta(\psi) \ll 1$, we transform to the new set of variables $\left\{\nu_{i}, e_{i}, p_{i}, i=1,2\right\}$, where $\nu_{1}=x_{1}+x_{2}+$ $x_{3}, e_{1}=\left(x_{1}-x_{3}\right) / 2 \nu_{1}$ and $p_{1}=\left(x_{1}-2 x_{2}+x_{3}\right) / 2 \nu_{1}$. The variables $\left(\nu_{2}, e_{2}, p_{2}\right)$ are defined as a function of the eigenvalues $y_{i}$ in a similar way. $e_{i}$ and $p_{i}$ are the shear ellipticity and prolateness, respectively. Our choice of ordering impose the constraints $e_{i} \geq 0$ and $-e_{i} \leq p_{i} \leq e_{i}$.

The cross-correlation function $\zeta_{i j}(r)$, or two-point connected moment of the shear eigenvalues $x_{i}$ and $y_{j}$ is defined as

$$
\left\langle x_{i}\right\rangle\left\langle y_{j}\right\rangle+\zeta_{i j}(r)=\int \mathrm{d}^{3} x \mathrm{~d}^{3} y P(x, y ; r) x_{i} y_{j} .
$$

The integration over the variables $p_{i}$ and $e_{i}$ is straightforward to second order in $\beta$. In this respect, notice that the volume measure $\mathrm{d}^{3} x$ and the Vandermonde determinant $\Delta(x)$ are $\mathrm{d}^{3} x=(2 / 3) \nu_{1}^{2} \mathrm{~d} \nu_{1} \mathrm{~d} e_{1} \mathrm{~d} p_{1}$ and $\Delta(x)=2 \nu_{1}^{3} e_{1}\left(e_{1}^{2}-p_{1}^{2}\right)$, respectively. Furthermore, with the help of the series expansion (23) about $\beta=0$, the average over the relative orientation can be reduced to

$$
{ }_{0} F_{0}^{(3)} \approx 1+\frac{\beta}{3} \nu_{1} \nu_{2}+\frac{\beta^{2}}{9} \nu_{1}^{2} \nu_{2}^{2}\left[\frac{1}{2}+\frac{2}{5}\left(3 e_{1}^{2}+p_{1}^{2}\right)\left(3 e_{2}^{2}+p_{2}^{2}\right)\right]
$$

in the aforementioned coordinates. The integration over the peak heights $\nu_{1}$ and $\nu_{2}$ is then easily performed and gives

$$
\zeta_{i i}(r)=\frac{1}{9} \psi(r)+\frac{1}{10}\left\langle x_{i}\right\rangle^{2} \psi^{2}(r)
$$

at second order for all three eigenvalues. To derive this result, we have used the following expressions for the mean of the shear eigenvalues $[24,26]$,

$$
\left\langle x_{1}\right\rangle=\left\langle x_{3}\right\rangle=\frac{3}{\sqrt{10 \pi}},\left\langle x_{2}\right\rangle=0 .
$$

Hence, there is no second order contribution to $\zeta_{22}(r)$. It is worthwhile remembering that the variance $\left\langle x_{i}^{2}\right\rangle$ of each eigenvalue is

$$
\left\langle x_{1}^{2}\right\rangle=\left\langle x_{3}^{2}\right\rangle=\frac{13 \pi-27}{30 \pi},\left\langle x_{2}^{2}\right\rangle=\frac{2}{15},
$$




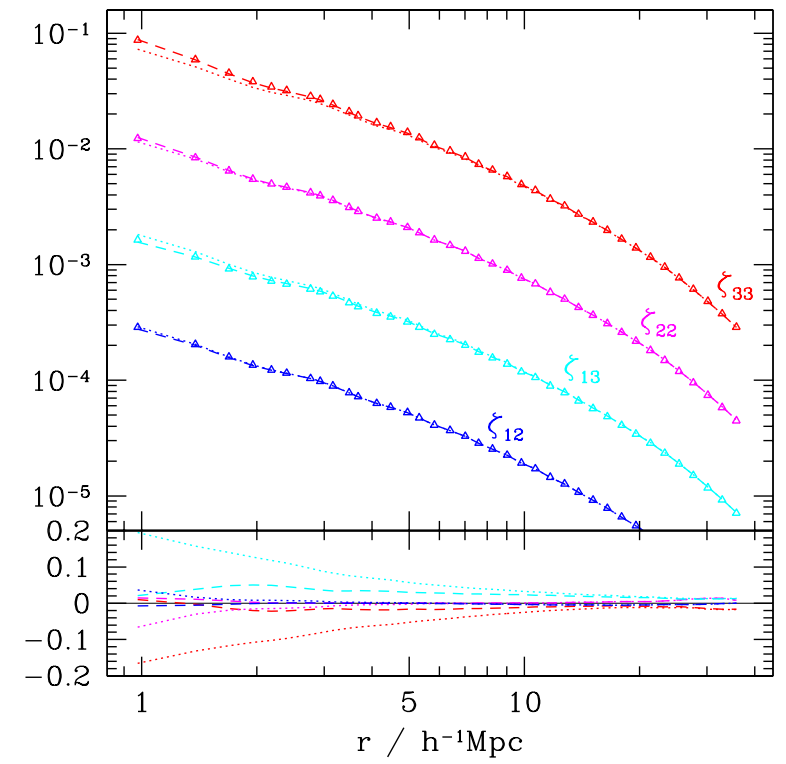

FIG. 2: A comparison between a measurement of the autoand cross-correlation of the shear eigenvalues from realizations of Gaussian random fields (symbols) and our analytic approximations (curves). All the correlations but $\zeta_{11}$ have been shifted vertically for clarity. On large scale, the correlations asymptote to the exact result $\psi(r) / 9$ shown as the dotted curve. On small scale however, $\bar{\zeta}_{i j}(r)$ (as defined in eqs. 30 and 36) provides a better fit to the measurement than the linear result. The bottom panel shows the fractional error. Notice that the density correlation $\psi(r)$ used for the comparison is calculated from the numerical realizations to account for the missing power at small and large scales.

close to the value of $1 / 9$. This suggests that the correlation functions $\zeta_{i i}(r)$ are well approximated by $\psi(r) / 9$ on all scales. This is not entirely surprising since, in the case where the correlations between eigenvalues are equal, the constraint $\sum_{i, j} \zeta_{i j}(r)=\psi(r)$ would imply $\zeta_{i j}(r)=\psi(r) / 9$.

In order to verify this assumption, we have generated random realizations of the potential field $\Phi(\boldsymbol{q})$ for the $\Lambda \mathrm{CDM}$ cosmology considered here on a $256^{3}$ mesh of size $250 \mathrm{~h}^{-1} \mathrm{Mpc}$. The eigenvalues of the shear tensor are computed on the same grid using standard FFT techniques. More precisely, the Fourier modes of the shear are computed using the relation $\xi_{i j}(\boldsymbol{k})=k_{i} k_{j} \Phi(\boldsymbol{k}) / \sigma$. Once $\xi_{i j}(\boldsymbol{k})$ is Fourier-transformed back, the shear eigenvalues $x_{i}$ as well as the density $\nu=x_{1}+x_{2}+x_{3}$ are calculated at each grid point. Lastly, after having checked that the measured variances $\left\langle x_{i}^{2}\right\rangle$ match well the analytic expectation (32), we calculate correlation functions of the shear eigenvalues. Note that the gravitational potential is smoothed on scale $R_{S}=1 h^{-1} \mathrm{Mpc}$.

In Fig 2, the correlations $\zeta_{22}(r)$ and $\zeta_{33}(r)$ (recall that $\left.\zeta_{11}=\zeta_{33}\right)$ averaged over the realizations are shown as empty symbols. These measurements are compared to the asymptotic scaling (30) and to the following analytic estimates

$$
\begin{aligned}
& \bar{\zeta}_{11}(r)=\frac{1}{9} \psi(r)+\left(\frac{87}{270}-\frac{9}{10 \pi}\right) \psi^{2}(r) \\
& \bar{\zeta}_{22}(r)=\frac{1}{9} \psi(r)+\frac{1}{45} \psi^{3}(r),
\end{aligned}
$$

which are designed to asymptote to the variances given in equation (32). The density correlation function $\psi$ measured from the simulations is used for the evaluation of those theoretical expressions. Fig 2 clearly demonstrates that, while the linear approximation $\psi / 9$ is in excellent agreement with the measurements in the asymptotic regime, it deviates at least 10 per cent at small distance, $r \leq 3 h^{-1} \mathrm{Mpc}$. By contrast, $\bar{\zeta}_{i i}(r)$ as defined above achieves a fractional error no larger than 2 per cent for separations less than $\sim 30 h^{-1} \mathrm{Mpc}$.

These results are readily extended to the crosscorrelations $\zeta_{i j}, i \neq j$. Proceeding in a similar way, $\zeta_{i j}(r)$ can be rearranged as

$$
\zeta_{i j}(r)=\frac{1}{9} \psi(r)+\frac{1}{10}\left\langle x_{i}\right\rangle\left\langle y_{j}\right\rangle \psi^{2}(r)
$$

to second order in $\psi(r)$. Estimating the cross-correlations at zero lag $\zeta_{i j}(0)=\left\langle x_{i} x_{j}\right\rangle$ from the theoretical probability distributions $P\left(x_{i}, x_{j}\right)$ proves difficult. A numerical integration gives the following hypothesized rational forms

$$
\left\langle x_{1} x_{2}\right\rangle=\left\langle x_{2} x_{3}\right\rangle=\frac{1}{10},\left\langle x_{1} x_{3}\right\rangle=\frac{27-6 \pi}{30 \pi},
$$

for which the constraint $\left\langle\left(x_{1}+x_{2}+x_{3}\right)^{2}\right\rangle=1$ is satisfied. This motivates the interpolation formulae

$$
\begin{aligned}
& \bar{\zeta}_{13}(r)=\frac{1}{9} \psi(r)-\left(\frac{14}{45}-\frac{9}{10 \pi}\right) \psi^{2}(r) \\
& \bar{\zeta}_{12}(r)=\bar{\zeta}_{23}(r)=\frac{1}{9} \psi(r)-\frac{1}{90} \psi^{3}(r),
\end{aligned}
$$

which match reasonably well the large- and small-scale behavior of $\zeta_{i j}$ (Fig. 2). Finite grid size effects may be responsible for the slight offset (roughly 2 per cent) of the cross-correlation $\zeta_{13}$ relative to the theoretical prediction.

Numerical investigations indicate that dark matter haloes do not form randomly in the initial conditions, but rather preferentially close to the peaks of the density field [40]. To assess the extent to which biasing affects our results, we adopt in a first approximation the usual critical density criterion issued from the spherical infall model. As first recognized in [41], the correlation function of regions lying above a certain density threshold $\nu$ is enhanced relative to that of the density correlation $\psi(r)$. Likewise, the correlations of shear eigenvalues restricted to regions with density larger than a given threshold $\nu$ are also amplified. On large scales and in the regime $\nu \gg 1$, we find

$$
\zeta_{i i}(r \mid>\nu) \approx\left(\frac{1}{3}+\nu\left\langle x_{i} \mid \nu\right\rangle\right)^{2} \psi(r),
$$




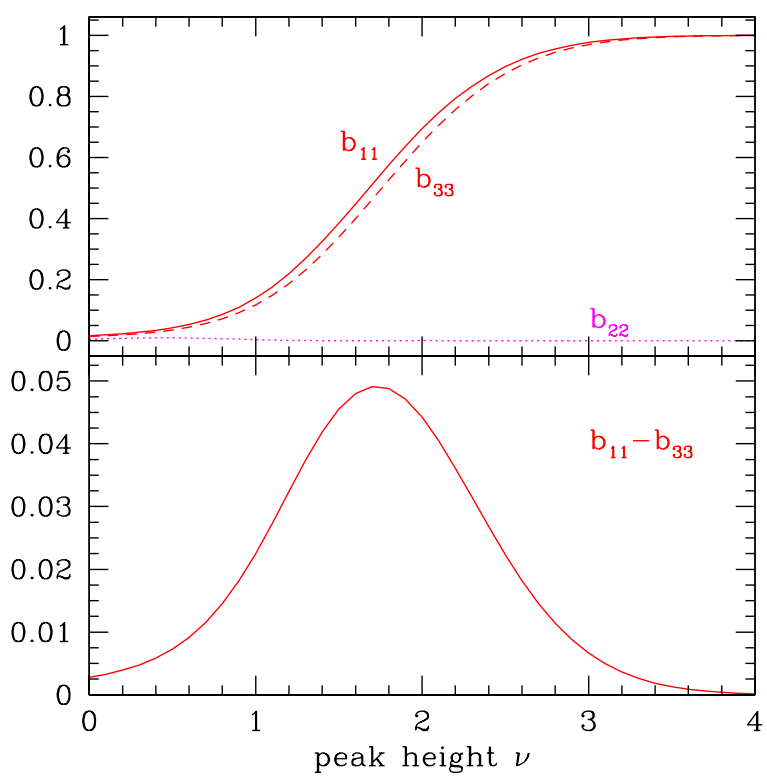

FIG. 3: Correction factors $b_{i i}(\Delta, \nu)$ (see text) as a function of the threshold height $\nu$ when the alignment of principal axes is restricted to those regions where all shear eigenvalues are positive. The bottom panel show the difference $b_{11}-b_{33}$, which demonstrates that the alignment between major axes is slightly stronger than the correlation of minor axes. The correlation of the intermediate axis is strongly suppressed over the whole range of peak height.

where the conditional average eigenvalue $\left\langle x_{i} \mid \nu\right\rangle$,

$$
\left\langle x_{i} \mid \nu\right\rangle=\left\langle x_{i}\right\rangle+\frac{\nu}{3},
$$

depends linearly on the peak height $\nu$. This should be compared to the correlation function of thresholded regions, which is $\xi(r \mid>\nu) \approx \nu^{4} \psi(r)$ in the same limit [41]. Hence, $\zeta_{i i}(r \mid>\nu)$ also exhibits the usual linear amplification factor $\nu^{4}$ of dense regions. It would be interesting to estimate the extent to which the large-scale bias varies when constraints are imposed on the shear eigenvalues. This calculation is postponed to a future work.

\section{B. Principal axes}

We now turn to the correlation of the shear principal axes. Since it is computationally expensive to measure such a correlation from numerical realizations (direct summation must be employed), we will only present an analytic estimate which is valid on large-scales. We also discuss its dependence on the peak height. Note that similar calculations for the correlation of angular momentum $L_{i} \propto \epsilon_{i j k} \xi_{j l} I_{l k}$, where $I_{l k}$ is the inertia tensor of some Lagrangian region, can be found in $[32,33]$ for instance.

Let $\hat{\boldsymbol{n}}_{i}$ designate the unit vector in the direction of the major, intermediate or minor axis of the shear. It is conventional to use

$$
\eta_{i j}(r)=\left\langle\left|\hat{\boldsymbol{n}}_{i}\left(\boldsymbol{q}_{1}\right) \cdot \hat{\boldsymbol{n}}_{j}\left(\boldsymbol{q}_{2}\right)\right|^{2}\right\rangle-\frac{1}{3}
$$

as a measure of the alignment between principal axes [42, 43], so that $\eta_{i j}(r)=0$ in the absence of any correlation.

We will only consider the correlations $\eta_{i i}$ since the calculation of $\eta_{i j}$ proceeds along similar lines. We parametrise the rotation matrix $\mathrm{R}$ in terms of the Euler angles $0 \leq \varphi, \psi \leq 2 \pi$ and $0 \leq \vartheta \leq \pi$. We adopt the $\mathrm{XYX}, \mathrm{YZY}$, and ZYZ convention when working out the correlation of major, intermediate and minor axes, respectively, so that $\cos \vartheta$ always is the cosine of the angle between the considered axes. The average is performed over the independent components of the shear tensor,

$$
\eta_{i i}(r)=\int \mathrm{d} \xi_{1} \mathrm{~d} \xi_{2}\left(\cos ^{2} \vartheta-\frac{1}{3}\right) P\left(\xi_{1}, \xi_{2} ; r\right)
$$

where, in the limit $r \gg 1$, the two-point probability distribution $P\left(\xi_{1}, \xi_{2} ; \boldsymbol{r}\right)$ reduces to eq. (17). The quadratic form $Q_{1}\left(\Lambda_{1}, \mathrm{R} \Lambda_{2} \mathrm{R}^{\top}\right)$ can be expanded in terms of the Wigner D-functions $\mathcal{D}_{m_{1}, m_{2}}^{l}, l$ being the index of the representation. These $3 \mathrm{D}$ harmonics generate irreducible representations of the three-dimensional rotation group and, therefore, form a complete orthogonal set of functions defined on $\mathrm{SO}(3)$ itself. Invariance under reflections implies that the quadrupole rotation matrices with $m=0, \pm 2$ appear in the harmonic decomposition of the quadratic form $Q_{1}$ as follows :

$$
\begin{aligned}
& Q_{1}\left(\Lambda_{1}, \mathrm{R} \Lambda_{2} \mathrm{R}^{\top}\right)=\frac{1}{2} \operatorname{tr} \Lambda_{1} \operatorname{tr} \Lambda_{2}+\frac{15 \epsilon_{-}}{8}\left[\mathcal{D}_{0,0}^{2}\right. \\
& -\sqrt{\frac{3}{2}} \epsilon_{1}\left(\mathcal{D}_{0,-2}^{2}+\mathcal{D}_{0,2}^{2}\right)-\sqrt{\frac{3}{2}} \epsilon_{2}\left(\mathcal{D}_{-2,0}^{2}+\mathcal{D}_{2,0}^{2}\right) \\
& \left.+\frac{3}{2} \epsilon_{1} \epsilon_{2}\left(\mathcal{D}_{-2,-2}^{2}+\mathcal{D}_{2,2}^{2}+\mathcal{D}_{-2,2}^{2}+\mathcal{D}_{2,-2}^{2}\right)\right] .
\end{aligned}
$$

The explicit form of these harmonics in the ZYZ representation is given in Table I. It is worth emphasizing that, apart from the "traceful" contribution $1 / 2 \operatorname{tr} \Lambda_{1} \operatorname{tr} \Lambda_{2}$, $Q_{1}\left(\Lambda_{1}, \mathrm{R} \Lambda_{2} \mathrm{R}^{\top}\right)$ depends on the three "shape" parameters $\epsilon_{-}, \epsilon_{1}$ and $\epsilon_{2}$ solely, because points on $\mathrm{SO}(3)$ truly have only three degrees of freedom. These parameters are given by $\epsilon_{-}=(1 / 3)\left(x_{13}+x_{23}\right)\left(y_{13}+y_{23}\right)$, $\epsilon_{1}=\left(x_{1}-x_{2}\right) /\left(x_{13}+x_{23}\right), \epsilon_{2}=\left(y_{1}-y_{2}\right) /\left(y_{13}+y_{23}\right)$, where $x_{i j}=x_{i}-x_{j}$ and $y_{i j}=y_{i}-y_{j}$. The alignment is simply $\eta_{i j}=2 / 3\left\langle\mathcal{D}_{0,0}^{2}\right\rangle$ with our parametrisation of the rotation matrix. Addition of angular momentum then yields

$$
\frac{1}{4} \int_{\mathrm{SO}(3)} \mathrm{dR}\left(\cos ^{2} \vartheta-\frac{1}{3}\right) Q_{1}\left(\Lambda_{1}, \mathrm{R} \Lambda_{2} \mathrm{R}^{\top}\right)=\frac{\epsilon_{-}}{16} .
$$

after averaging over the angular variables. Lastly, the integral over the volume measure $\mathrm{d}^{3} x \mathrm{~d}^{3} y$ is easily performed in the coordinate system $(\nu, e, p)$, where

$$
\epsilon_{-}= \begin{cases}\frac{1}{3} \nu_{1} \nu_{2}\left(3 e_{1}-p_{1}\right)\left(3 e_{2}-p_{2}\right) & \text { minor axis } \\ \frac{1}{3} \nu_{1} \nu_{2}\left(3 e_{1}+p_{1}\right)\left(3 e_{2}+p_{2}\right) & \text { major axis }\end{cases}
$$



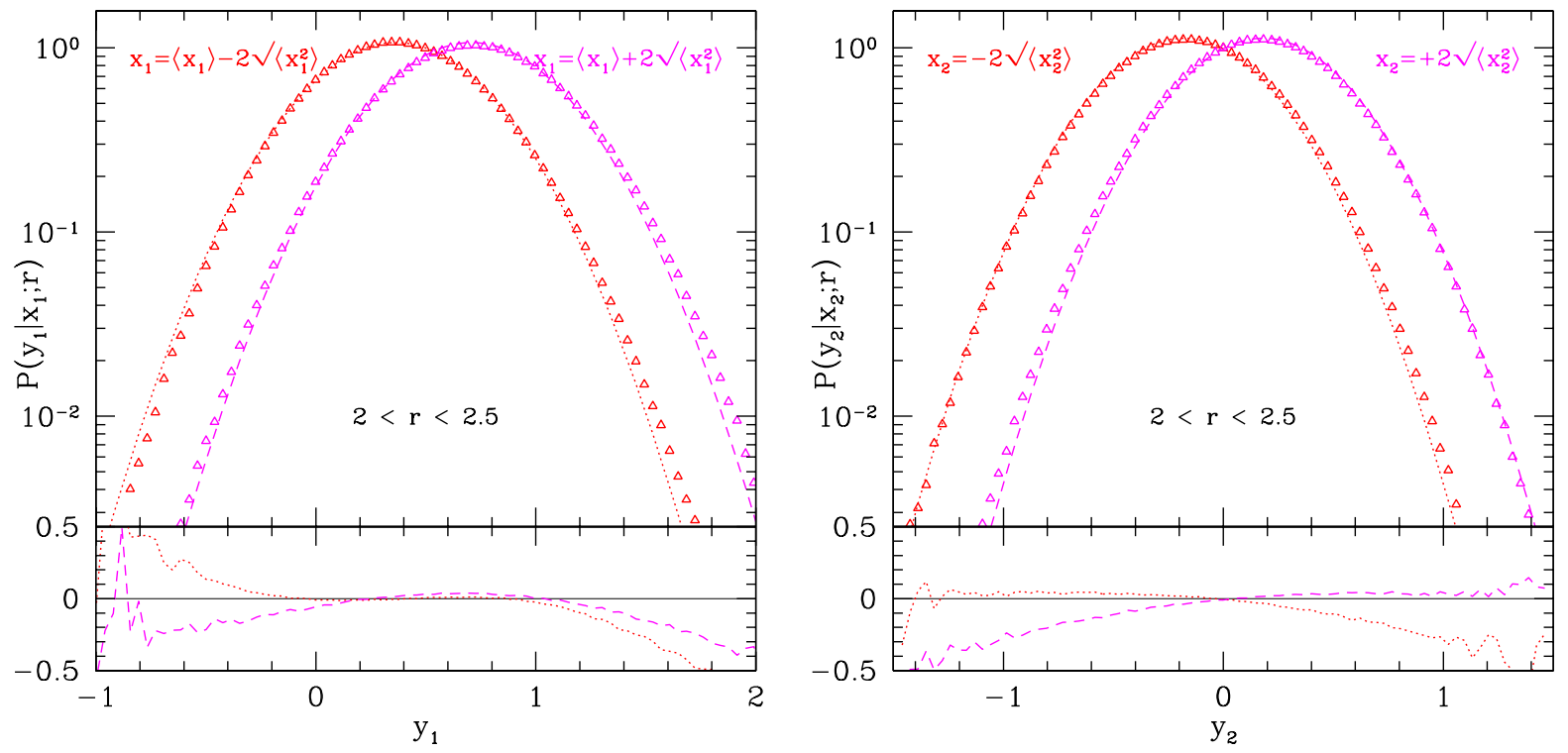

FIG. 4: A comparison between the conditional two-point probability distribution $P\left(y_{i} \mid x_{i} ; r\right)$ measured from realizations of Gaussian random fields and the Gaussian approximation eq. (51). Results are presented for the largest and intermediate eigenvalues only (left and right panels respectively). Symbols show the measurements of the conditional probability for a separation $2<r<2.5 h^{-1} \mathrm{Mpc}$ and for $x_{i}=\left\langle x_{i}\right\rangle \pm 2 \tilde{\sigma}_{i}$. The correlation $\zeta_{i i}(r)$ used in the analytic estimate shown as the dashed curve is directly calculated from the random realizations of the shear field. The bottom panels show the fractional error.

Let us choose $\nu_{1}=\nu_{2}=\nu$ for illustration purpose, and perform the integration over the domain defined by $e_{i} \geq 0$ and $\left|p_{i}\right| \leq e_{i}(i=1,2)$. We find that, in the large-scale limit $r \gg 1$, the alignment $\eta_{i i}(r \mid>\nu)$ of thresholded regions evaluates to

$$
\eta_{i i}(r \mid>\nu)=\frac{27}{20 \pi} \psi(r) \approx 0.43 \psi(r)
$$

regardless of the peak height and the axis under consideration (major and minor). This is unsurprising given the invariance of the integral over the asymmetry parameters under the reflection $p_{i} \rightarrow-p_{i}$. For the intermediate axis, a similar calculation yields $\epsilon_{-}=(4 / 3) \nu_{1} \nu_{2} p_{1} p_{2}$. This implies $\eta_{22}(r \mid>\nu)=0$ at leading order since the one-point probability $P(e, p \mid \nu)$,

$$
P(e, p \mid \nu)=\frac{1125}{\sqrt{10 \pi}} \nu^{5} e\left(e^{2}-p^{2}\right) e^{-\frac{5}{2} \nu^{2}\left(3 e^{2}+p^{2}\right)},
$$

is symmetric about $p=0$.

Unlike the conditional correlation $\zeta_{i i}(r \mid>\nu)$ of shear eigenvalues, the alignment $\eta_{i i}(r \mid>\nu)$ between the shear principal axes is insensitive to the peak height. However, restricting the domain of integration to the region where all shear eigenvalues are positive, for instance, can introduce a dependence on the threshold height. Such a constraint naturally arises in models of structure formation to characterize the Lagrangian regions which collapse into dark matter haloes. The domain where the lowest eigenvalue is positive corresponds to the interior of the triangle bounded by $(e, p)=(0,0),\left(\frac{1}{4},-\frac{1}{4}\right)$ and $\left(\frac{1}{2}, \frac{1}{2}\right)$. The conditional correlation can be conveniently expressed as $b_{i i}(\Delta, \nu) \eta(r \mid>\nu)$, where $\eta(r \mid>\nu)=$ $27 / 20 \pi \psi(r)$ and $b_{i i}(\Delta, \nu)$ is a correction factor resulting from the restriction to the triangular domain $\lambda_{3}>0$. After some manipulation, we find

$$
\begin{aligned}
b_{11}(\Delta, \nu) & \approx\left\{1-\operatorname{Erf}\left(\frac{\nu}{\sqrt{2}}\right)^{-1}\left[\sqrt{\frac{2}{\pi}} \frac{5 \nu}{27} e^{-9 \nu^{2} / 8}\right.\right. \\
& +\frac{\sqrt{60}}{27} \operatorname{Erf}\left(\sqrt{\frac{15}{2}} \frac{\nu}{2}\right) e^{-9 \nu^{2} / 8}+\frac{34}{81} \operatorname{Erfc}\left(\frac{3 \nu}{2 \sqrt{2}}\right) \\
& \left.\left.+\frac{5 \sqrt{6}}{27} \operatorname{Erfc}(\sqrt{3} \nu)\right]\right\}^{2} \\
b_{22}(\Delta, \nu) & \approx\left\{\left[\sqrt{\frac{2}{\pi}} \frac{5 \nu}{27}-\frac{\sqrt{60}}{27} \operatorname{Erf}\left(\sqrt{\frac{15}{2}} \frac{\nu}{2}\right)\right]\right. \\
& \left.\times e^{-9 \nu^{2} / 8}+\frac{34}{81} \operatorname{Erfc}\left(\frac{3 \nu}{2 \sqrt{2}}\right)-\frac{1}{\sqrt{6}} \operatorname{Erfc}(\sqrt{3} \nu)\right\}^{2} \\
& \times \operatorname{Erf}\left(\frac{\nu}{\sqrt{2}}\right)^{-2} \\
b_{33}(\Delta, \nu) & \approx\left\{1-\operatorname{Erf}\left(\frac{\nu}{\sqrt{2}}\right)^{-1}\left[\sqrt{\frac{2}{\pi}} \frac{10 \nu}{27} e^{-9 \nu^{2} / 8}\right.\right. \\
& \left.\left.+\frac{68}{81} \operatorname{Erfc}\left(\frac{3 \nu}{2 \sqrt{2}}\right)+\frac{\sqrt{6}}{54} \operatorname{Erfc}(\sqrt{3} \nu)\right]\right\}^{2}
\end{aligned}
$$

These expressions are plotted in the upper panel of Fig. 3 as a function of the threshold height. Clearly, the align- 
TABLE I: Quadrupole Wigner D-functions $\mathcal{D}_{m_{1}, m_{2}}^{2}(\varphi, \vartheta, \psi)$ in the ZYZ representation. Harmonics with $m_{1}, m_{2}= \pm 1$ are not shown since they are not needed for the present analysis.

\begin{tabular}{llll}
\hline \hline & $m_{2}=-2$ & $m_{2}=0$ & $m_{2}=2$ \\
\hline$m_{1}=-2$ & $\frac{1}{4}(1+\cos \vartheta)^{2} e^{2 i \varphi+2 i \psi}$ & $\sqrt{\frac{3}{8}} \sin ^{2} \vartheta e^{2 i \varphi}$ & $\frac{1}{4}(1-\cos \vartheta)^{2} e^{2 i \varphi-2 i \psi}$ \\
$m_{1}=0$ & $\sqrt{\frac{3}{8}} \sin ^{2} \vartheta e^{2 i \psi}$ & $\frac{1}{2}\left(3 \cos ^{2} \vartheta-1\right)$ & $\sqrt{\frac{3}{8}} \sin ^{2} \vartheta e^{-2 i \psi}$ \\
$m_{1}=2$ & $\frac{1}{4}(1-\cos \vartheta)^{2} e^{-2 i \varphi+2 i \psi}$ & $\sqrt{\frac{3}{8}} \sin ^{2} \vartheta e^{-2 i \varphi}$ & $\frac{1}{4}(1+\cos \vartheta)^{2} e^{-2 i \varphi-2 i \psi}$ \\
\hline \hline
\end{tabular}

ment between major and minor axes is strongly suppressed when $\nu \leqslant 2$. The correction factors $b_{i i}(\Delta, \nu)$ are always less than one, to which they asymptote in the limit of large threshold $\nu$. Furthermore, in the range $\nu \sim 1-3$, we have $b_{11} \gtrsim b_{33}$ so that the correlation between major axes is stronger by a few per cent. Shown in the lower panel of Fig. 3 is the difference $b_{11}-b_{33}$. It is maximal when the integral of the probability distribution $P(e, p \mid \nu)$ over the triangle is about one half. This occurs when the mean ellipticity $\langle e \mid \nu\rangle=3 /(\sqrt{10 \pi} \nu)$ roughly is $1 / 4-1 / 2$, i.e. when $\nu \approx 2$. Restricting the integration domain to $\lambda_{3}>0$ thus induces a non-zero, albeit small, correlation between intermediate axes. $\eta_{22}(r \mid>\nu)$ does not exceed $\sim 0.01 \psi$, a value reached when $\nu \sim 0.5$. We emphasize that the alignment between the principal axes of the shear field is in all cases proportional to the density correlation on large scales. This is consistent with the findings of $[33,44]$.

We have shown that the initial alignment of the shear principal axes is significant only for those regions which collapse into haloes of mass $M \gtrsim M_{\star}$. We will, nevertheless, not extend the discussion to the statistics of dark matter halo and galaxy shapes as it is unclear to which extent the correlations detected in N-body simulations of CDM cosmologies [17], or in galaxy surveys [16], reflect the large-scale alignment of the initial shear. Nonlinear effects, such as anisotropic accretion or relaxation following collapse, could plausibly enhance or erase the large-scale coherence of the primordial tidal field [14, 49]. Indeed, even in the artificial case where all dark matter haloes are perfectly aligned would the alignment and clustering of galaxies be negligibly affected [45]. We conclude by noticing that primordial non-gaussianities characterized by a local mapping of the form $\phi_{\mathrm{NG}}=\phi-f_{\mathrm{NL}}\left(\phi^{2}-\left\langle\phi^{2}\right\rangle\right) \quad[46,47,48]$ would not affect the correlation of principal axes.

\section{A GAUSSIAN APPROXIMATION}

\section{A. Shear eigenvalues}

Explicit expressions for the one-point probability distribution $P\left(x_{i}\right)$ of the individual shear eigenvalues $x_{i}$ can be found in $[24,26]$. It is worthwhile noticing that , although the variables $x_{i}(\boldsymbol{q})$ are not Gaussian random

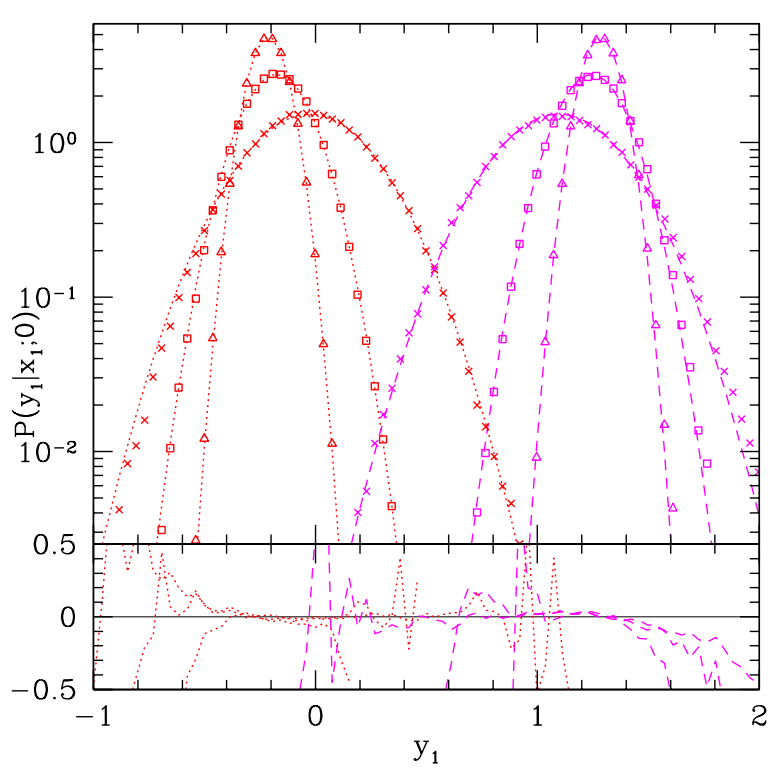

FIG. 5: The conditional probability density $P\left(y_{1} \mid x_{1} ; 0\right)$ evaluated at a single Lagrangian position but at two different smoothing lengths $R_{1}$ and $R_{2}$. The triangles, squares and crosses show $P\left(y_{1} \mid x_{1} ; 0\right)$ measured from random realizations of the potential smoothed on scale $R_{2}=2.5,3$ and $5 h^{-1} \mathrm{Mpc}$, respectively, while keeping $R_{1}=2 h^{-1} \mathrm{Mpc}$ fixed. The dotted and dashed curves indicate the Gaussian approximation when $x_{1}=\left\langle x_{1}\right\rangle+2 \tilde{\sigma}_{1}$ and $\left\langle x_{1}\right\rangle-2 \tilde{\sigma}_{1}$, respectively. The bottom panel shows the fractional error.

fields, their one-point probability distributions are very close to Gaussian. More precisely, $P\left(x_{2}\right)$ is indeed (fortuitously) exactly Gaussian, whereas the probability densities $P\left(x_{1}\right)$ and $P\left(x_{3}\right)$ show a small positive skewness,

$$
\text { skewness }=\frac{3^{3 / 2}(54-17 \pi)}{(13 \pi-27)^{3 / 2}} \approx 0.060,
$$

which reflects the fact that the large tail of these distribution is slightly more pronounced.

To assess the extent to which two-point statistics of the shear eigenvalues deviate from Gaussianity, we have measured the conditional, two-point probability density $P\left(y_{i} \mid x_{i} ; r\right)$ from random realizations of the potential field. Results are presented in Fig. 4 (symbols) for a separation 
in the range $2<r<2.5 h^{-1} \mathrm{Mpc}$, and for two particular values of $x_{i}: x_{i}=\left\langle x_{i}\right\rangle \pm 2 \tilde{\sigma}_{i}$ where $\tilde{\sigma}_{i}^{2} \equiv\left\langle x_{i}^{2}\right\rangle$ in what follows. These measurements are compared to the following Gaussian conditional distribution

$$
\begin{aligned}
P\left(y_{i} \mid x_{i} ; r\right)= & \frac{\tilde{\sigma}_{i}}{\sqrt{2 \pi\left(\tilde{\sigma}_{i}^{4}-\zeta_{i i}^{2}\right)}} \\
& \times \exp \left[-\frac{\tilde{\sigma}_{i}^{2}\left(\tilde{x}_{i}^{2}+\tilde{y}_{i}^{2}\right)-2 \zeta_{i i} \tilde{x}_{i} \tilde{y}_{i}}{2\left(\tilde{\sigma}_{i}^{4}-\zeta_{i i}^{2}\right)}+\frac{\tilde{x}_{i}^{2}}{2 \tilde{\sigma}_{i}^{2}}\right],
\end{aligned}
$$

where, for shorthand convenience, we have introduced the variable $\tilde{x}_{i} \equiv x_{i}-\left\langle x_{i}\right\rangle$. This conditional probability is shown as the dashed curves. Figure 4 nicely demonstrates the validity of the Gaussian approximation down to scales of the order of the grid resolution (about $1 h^{-1} \mathrm{Mpc}$ ). We also note that, in the distribution $P\left(y_{1} \mid x_{1} ; r\right)$, the skewness increases by $\sim 50$ per cent to reach $\approx 0.089$ at separation $r \sim 2 h^{-1} \mathrm{Mpc}$ whereas in $P\left(y_{2} \mid x_{2} ; r\right)$, the skewness is approximately 0.016 at the same distance.

Thus far, we have restricted the comparison to the case in which the shear at Lagrangian positions $\boldsymbol{q}_{1}$ and $\boldsymbol{q}_{2}$ is smoothed at the same length $R_{S}$. To further assess the validity of the Gaussian approximation in the limit $\zeta_{i i} \approx \tilde{\sigma}_{i}^{2}$, we take advantage of the fact that the joint distribution of the shear eigenvalues, evaluated at a single Lagrangian position $\boldsymbol{q}_{1}=\boldsymbol{q}_{2}$ and two different smoothing lengths $R_{1}$ and $R_{2}$, also has the functional form of equation (19), with $\psi=\gamma$ [28]. Therefore, despite the limitation arising from the finite grid spacing, we can nevertheless probe the strongly correlated regime by studying the conditional probability density $P\left(y_{i} \mid x_{i} ; 0\right)$ in the limit $R_{1} \approx R_{2}$. For illustration, let us consider the largest eigenvalue. We set $R_{1}=2 h^{-1} \mathrm{Mpc}$ and take $R_{2}=2.5,3$ and $5 h^{-1} \mathrm{Mpc}$. The cross-correlation $\zeta_{11}$ at two different smoothing scales, computed following the procedure outlined in $\S \mathrm{IV}$, yields $\tilde{\sigma}_{1}^{2}=0.143,0.136$ and 0.108 [51], respectively. These values are used for the evaluation of the conditional density (51). As seen from Fig. 5, there is, as before, a remarkably good agreement between $P\left(y_{1} \mid x_{1} ; 0\right)$ measured from random realizations of the potential $\Phi$ and the conditional bivariate Gaussian eq. (51), except for the very tails of the distributions. This strongly suggests that Gaussian statistics are an accurate approximation to the statistics of shear eigenvalues at all separations and smoothing scales.

\section{B. Gradients of the shear eigenvalues}

The derivatives of any Gaussian random field $X(\boldsymbol{q})$ with respect to the coordinate $\boldsymbol{q}$ also are Gaussian random fields since the differential operators $\partial / \partial q_{i}$ are linear. To ascertain how much the spatial derivatives of the shear eigenvalues deviate from Gaussianity, we have examined a number of correlation functions and conditional two-point statistics, focusing on the first derivatives $\partial_{i} x_{1}$ of the largest eigenvalue. This isotropic vector field has

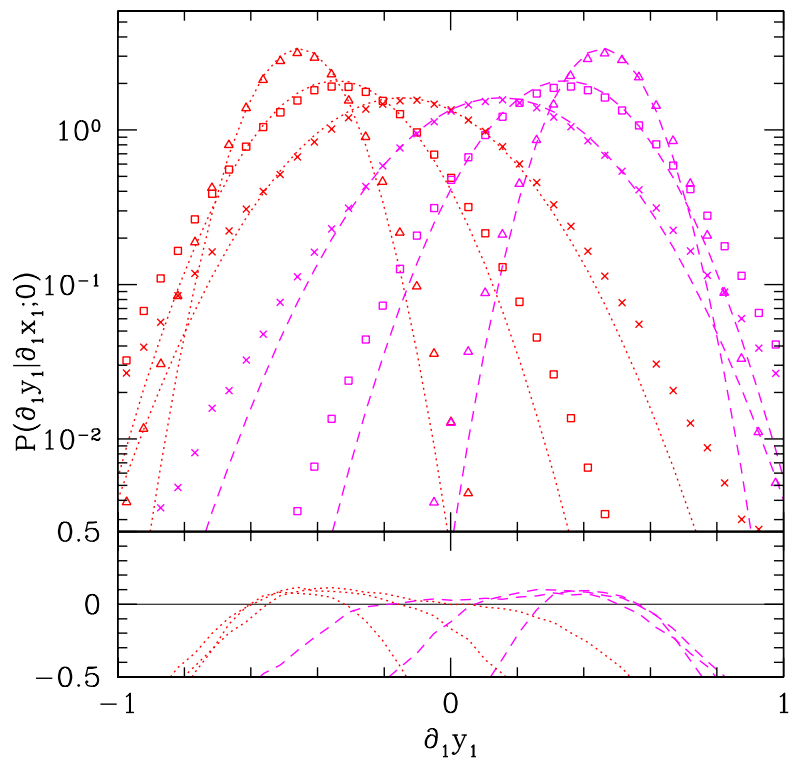

FIG. 6: Same as Fig. 5 but showing the conditional probability density $P\left(\partial_{1} \tilde{y}_{1} \mid \partial_{1} \tilde{x}_{1} ; 0\right)$. Deviations from the bivariate Gaussian are more pronounced here than in the conditional distribution $P\left(y_{1} \mid x_{1} ; 0\right)$.

a covariance tensor of the form

$$
\left\langle\partial_{i} x_{1} \partial_{j} y_{1}\right\rangle=\Sigma_{1}(r) \hat{r}_{i} \hat{r}_{j}+\Sigma_{2}(r) \delta_{i j} .
$$

When $\zeta_{11}$ is known analytically, exact expressions can be derived fairly easily using the relation $\left\langle\partial_{i} x_{1} \partial_{j} y_{1}\right\rangle=$ $-\partial_{i} \partial_{j} \zeta_{11}(r)$. For instance, the approximation (33) gives

$$
\begin{aligned}
& \left\langle\partial_{i} x_{1} \partial_{j} y_{1}\right\rangle \approx\left(\frac{1}{9}+c \psi^{2}\right) \delta_{i j} \\
& +\left[2 c \psi^{\prime 2}+\left(\frac{1}{9}+2 c \psi\right)\left(-\frac{\psi^{\prime}}{9}+\psi^{\prime \prime}\right)\right] \hat{r}_{i} \hat{r}_{j},
\end{aligned}
$$

where $c=87 / 270-9 / 10 \pi$ and a prime denotes a derivative with respect to $r$. Let us introduce the dimensionless variable $\partial_{i} \tilde{x}_{1} \equiv\left(\sigma_{0} / \sigma_{1}\right) \partial_{i} x_{1}$, where the $\sigma_{j}$ generally are the spectral moments of the density field [23],

$$
\sigma_{j}^{2} \equiv \int_{0}^{\infty} \operatorname{dln} k k^{2 j} \Delta_{\delta}^{2}(k) .
$$

Using equation (53) as an analytic estimate of the correlation $\left\langle\partial_{i} \tilde{x}_{1} \partial_{i} \tilde{y}_{1}\right\rangle$, we find a variance

$$
\left\langle\partial_{i} \tilde{x}_{1}^{2}\right\rangle=\frac{1}{3}\left(\frac{34}{45}-\frac{9}{5 \pi}\right) \approx 6.087 \times 10^{-2},
$$

somewhat 10 per cent smaller than the value of $\approx 6.60 \times$ $10^{-2}$ measured from the random realizations with $R_{S}$ in the range $2-5 h^{-1} \mathrm{Mpc}$. The agreement can be improved by adding higher order terms in the truncated expansion eq. (33). 
The conditional probability $P\left(\partial_{1} \tilde{y}_{1} \mid \partial_{1} \tilde{x}_{1} ; 0\right)$ is shown in Fig. 6 for three different smoothing lengths $R_{1} \approx R_{2}$ (as in Fig 5). Cross-correlations coefficients and variances are computed from the random realizations. Clearly, although deviations from Gaussianity are more pronounced than in the conditional two-point statistics of the eigenvalues discussed above, the agreement is still reasonable. However, we have found that it worsens significantly for the second derivative. In spite of this limitation, it would be valuable to assess whether the Gaussian approximation provides a reliable description of the statistics of extrema of the shear eigenvalues, since the latter play a particular role in non-spherical collapse models [9].

\section{CONCLUSION}

We have explored the statistical correlation that arises in Gaussian initial conditions between the properties of the linear tidal shear $\partial_{i} \partial_{j} \Phi$ at two distinct positions, thereby extending the work of $[23,24,26,27,28]$. In Section §III, using asymptotic expansions, we derived exact closed form expressions for the joint distribution of shear components and shear eigenvalues as a function of the Lagrangian separation. These results were applied to study the large distance asymptotics of the correlation function of the shear eigenvalues and the shear principal axes. In Section $§ I V$, we presented interpolation formulae that accurately match the large- and small-scale behavior of the correlation of shear eigenvalues measured from random realizations of the gravitational potential. We also found that the alignment of the shear principal axes of thresholded regions is insensitive to the threshold height. However, restricting the correlation to regions where all three eigenvalues are positive, introduces a dependence on the threshold density, which manifests itself as a strong suppression of the alignment for peak height less than $\nu \sim 1$. We emphasize that all these correlations are proportional to the density correlation on large scale.

In Section $\S \mathrm{V}$, we showed that the two-point statistics of the shear eigenvalues closely follow the Gaussian statistics regardless of the separation and the smoothing length. Although we have not formally established that Gaussian multivariates comply with measurements of the $n$-point distributions of shear eigenvalues, we speculate that the Gaussian approximation also holds for these multi-point distributions. Under this assumption, it should be fairly straightforward to apply the techniques and results obtained for Gaussian density fields to the shear eigenvalues.

Gaussian statistics provides also a reasonable description of a number of conditional probability densities involving first derivatives of the shear eigenvalues. Note, however, that the agreement worsens noticeably for the second derivatives. This caveat notwithstanding, a Gaussian approximation should be adequate to understand, at least qualitatively, the clustering of extrema of the shear eigenvalues for instance. The mathematical framework laid down by [23] appears well suited for such a study.
Our results can also be applied to the description of large-scale structures using the cosmic web approach based on the ellipsoidal collapse [8, 50]. In light of our analysis, conditional multivariate Gaussian describing the joint distribution of the density, displacement field and shear could easily be written down. As recognized in [8], these statistics will prove useful for quantifying the properties of the mildly nonlinear fluctuations, which evolve into the network of clusters, filaments and walls observed in the recent $2 \mathrm{dF}$ and SDSS galaxy surveys [1]. Constraints on the tidal shear could also be included in topological measures such as Minkowski functionals, in an attempt to study the effect of non-spherical infall on the morphology of the primeval large-scale structures.

\section{Acknowledgments}

We acknowledge support by the Swiss National Foundation under contract 200021-116696/1.

\section{APPENDIX A: AVERAGING OVER THE RELATIVE ORIENTATIONS}

The integral (22) over the relative orientation of the principal axis frames can be expressed as a hypergeometric function with the symmetric $3 \times 3$ matrices $\beta \Lambda_{1}$ and $\Lambda_{2}$ as argument,

$$
{ }_{0} F_{0}^{(3)}\left(\beta \Lambda_{1}, \Lambda_{2}\right)=\sum_{k=0}^{\infty} \frac{\beta^{k}}{k !} \sum_{\kappa} \frac{C_{\kappa}\left(\Lambda_{1}\right) C_{\kappa}\left(\Lambda_{2}\right)}{C_{\kappa}(\mathrm{I})}
$$

Here $\sum_{\kappa}$ designates summation over all partitions $\kappa \vdash$ $k$ of $k$, namely, over the ordered sequence of integer $\left(k_{1}, k_{2}, \ldots, k_{n}\right)$ such that $k_{1} \geq k_{2} \geq \cdots \geq k_{n} \geq 0$ and $\sum k_{i}=k . C_{\kappa}(\mathrm{X})$ are the zonal polynomials of the matrix X. They satisfy the relation $(\operatorname{trX})^{k}=\sum_{\kappa} C_{\kappa}(\mathrm{X})$. We emphasize that, despite the use of matrix notation here, $C_{\kappa}(\mathrm{X})$ is a function of the eigenvalues of $\mathrm{X}$ solely and could thus be written as $C_{\kappa}(x)$ for example.

The zonal polynomial can be expressed in terms of the monomial symmetric functions $m_{\kappa}(x)$. When $k=2$ for instance, there are two zonal polynomials corresponding to the partitions $(2)$ and $(1,1)$ of $2, C_{(2)}=m_{(2)}(x)+$ $2 / 3 m_{(1,1)}(x)$ and $C_{(1,1)}=4 / 3 m_{(1,1)}$, where

$$
\begin{aligned}
m_{(2)}(x) & =x_{1}^{2}+x_{2}^{2}+x_{3}^{2} \\
m_{(1,1)}(x) & =x_{1} x_{2}+x_{1} x_{3}+x_{2} x_{3} .
\end{aligned}
$$

The value of these zonal polynomial at $\mathrm{I}$ is $C_{(2)}(\mathrm{I})=5$ and $C_{(1,1)}(\mathrm{I})=4$. There is a recurrence relation between the coefficients of $m_{\kappa}(x)$ that determines $C_{\kappa}(\mathrm{X})$ uniquely once the coefficient of $m_{(k)}$ is given [37, 38]. Note also that the functions $m_{\kappa}(x)$ can be written in terms of the traces of power of $\mathrm{X}, \operatorname{trX}^{k}$ with $k, l=0,1, \ldots$ We refer the reader to [37] for further details. 
[1] M. Colless, et al., ArXiv Astrophysics eprint:astro-ph/0306581 (2003); J.K. AdelmanMcCarthy, et al., ArXiv Astrophysics eprint:astroph/0707.3413 (2007).

[2] C. Park, Mon. Not. R. Astron. Soc. , 242, 59 (1990); A. Nusser, A. Dekel, Astrophys. J. , 362, 14 (1990); D.H. Weinberg, J.E. Gunn, Mon. Not. R. Astron. Soc. , 247, 260 (1990); E. Bertschinger, J.M. Gelb, Comp. Phys. , 5, 164 (1991); R. Cen, J.P. Ostriker, Astrophys. J. , 417, 415 (1993); A. Jenkins, et al., Astrophys. J. , 499, 20 (1998); V. Springel, et al., Nature (London) , 435, 629 (2005); S. Gottlöber, G. Yepes, Astrophys. J. , 664, 117 (2007).

[3] J.E. Gunn, J.R. Gott III, Astrophys. J. , 176, 1 (1972).

[4] W.H. Press, P. Schechter, Astrophys. J. , 187, 425 (1974); J.R. Bond, S. Cole, G. Efstathiou, N. Kaiser, Astrophys. J. , 379, 440 (1991); C. Lacey, S. Cole, Mon. Not. R. Astron. Soc., 262, 627 (1993); H.J. Mo, S.D.M. White, Mon. Not. R. Astron. Soc., 282, 347 (1996); R.K. Sheth, G. Tormen, Mon. Not. R. Astron. Soc. , 308, 119 (1999).

[5] P.J.E Peebles, The Large-Scale Structure of the Universe (Princeton University Press, 1980).

[6] J.R.III Gott, A.L. Melott, M. Dickinson, Astrophys. J. , 306, 341 (1986); K.R. Mecke, T. Buchert, H. Wagner, Astron. Astrophys. , 288, 697 (1994); T. Matusbara, Astrophys. J. , 434, 43L (1994); J. Schmalzing, T. Buchert, Astrophys. J. , 482, L1 (1997); M. Kerscher, et al., Mon. Not. R. Astron. Soc., 284, 73 (1997); M. Kerscher, Lecture Notes in Physics, 554, 36 (2000). L. Gleser, A. Nusser, B. Ciardi, V. Desjacques, Mon. Not. R. Astron. Soc. , 370, 1329 (2006); J.R.III Gott, et al., Astrophys. J. , 675, 16 (2008).

[7] V. Sahni, B.S. Sathyaprakash, S.F. Shandarin, Astrophys. J. , 495, L5 (1998); S. Colombi, D. Pogosyan, T. Souradeep T., Phys. Rev. Lett. , 85, 5515 (2000); H. Hanami, Mon. Not. R. Astron. Soc. , 327, 721 (2001); E. Platen, R. van de Weygaert, B.J.T Jones, Mon. Not. R. Astron. Soc. , 380, 551 (2007); M.A. Aragón-Calvo, B.J.T. Jones, R. van de Weygaert, J.M. van der Hulst, Astron. Astrophys. , 474, 315 (2007); T. Sousbie, C. Pichon, S. Colombi, D. Novikov, D. Pogosyan, Mon. Not. R. Astron. Soc. , 383, 1655 (2008).

[8] J.R. Bond, L. Kofman, D. Pogosyan, Nature (London), 380, 603 (1996).

[9] Y.B. Zeldovich, Astron. Astrophys. , 5, 84 (1970); V.I. Arnold, S.F. Shandarin, Y.B. Zeldovich, Geophys. Astrophys. Fluid Dyn. , 20, 111 (1982); S.D.M. White, Astrophys. J. , 286, 38 (1984); Y. Hoffman, Astrophys. J. , 308, 493 (1986); L. Kofman, D. Pogosyan, S.F. Shandarin, A.L. Melott, Astrophys. J. , 393, 437 (1992); R. van de Weygaert, A. Babul, Astrophys. J. , 425, L59 (1994); E. Bertschinger, B. Jain, Astrophys. J., 431, 486 (1994); A. Audit, J.-M. Alimi, Astron. Astrophys. , 315, 11 (1996).

[10] R.K. Sheth, G. Tormen, Mon. Not. R. Astron. Soc. , 329, 61 (2002).

[11] B. Binggeli, Astron. Astrophys., 107, 338 (1982).

[12] P.C. Argyres, E.J. Groth, P.J.E. Peebles, M.F. Struble, Astron. J., 91, 471 (1986).
[13] M.J. West, A. Dekel, A. Oemler, Astrophys. J. , 336, 46 (1989).

[14] M.J. West, Astrophys. J. , 347, 610 (1989).

[15] P. Catelan, M. Kamionkowski, R.D. Blandford, Mon. Not. R. Astron. Soc., 320, L7 (2001).

[16] A. Heavens, A. Refregier, C. Heymans, Mon. Not. R. Astron. Soc. , 319, 649 (2000).

[17] R.A.C. Croft, C.A. Metzler, Astrophys. J. , 545, 561 (2000).

[18] P.F. Hopkins, N.A. Bahcall, P. Bode, Astrophys. J. , 618, 1 (2005).

[19] O. Hahn, C. Porciani, C.C. Carollo, A. Dekel, Mon. Not. R. Astron. Soc., 375, 489 (2007).

[20] E. Platen, R. van de Weygaert, B.J.T. Jones, ArXiv Astrophysics eprint:astro-ph/0711.2480 (2007).

[21] R.J. Adler, The Geometry of Random Fields (Chichester:Wiley, 1981).

[22] J.A. Peacock, A.F. Heavens, Mon. Not. R. Astron. Soc. , 217, 805 (1985).

[23] J.M. Bardeen, J.R. Bond, N. Kaiser, A.S. Szalay, Astrophys. J. , 304, 15 (1986).

[24] A.G. Doroshkevich, Astrofizika, 3, 175.

[25] A.G. Doroshkevich, S.F. Shandarin, Soviet. Astr., 22, 653 (1978).

[26] J. Lee, S. Shandarin, Astrophys. J. , 500, 14 (1998).

[27] P. Catelan , C. Porciani, Mon. Not. R. Astron. Soc. , 323, 713 (2001).

[28] V. Desjacques, astro-ph/0707.4670 (2007).

[29] F. Bernardeau, Astrophys. J. , 427, 51 (1994).

[30] D. Pogosyan, J.R. Bond, L. Kofman, J. Wadsley, 1998, in Proceedings of the XIV IAP Colloquium, Wide Field Surveys in Cosmology, ed. Y. Mellier and S. Colombi (Paris: Editions Frontieres).

[31] Komatsu, et al., Astrophys. J. Suppl. , 148, 119 (2003); P. Creminelli, N. Alberto, L. Senatore, M. Tegmark, M. Zaldarriaga, JCAP, 06, 004 (2006); A. Yadav, B.D. Wandelt, ArXiv Astrophysics eprint:astroph/0712.1148 (2007); E. Komatsu, et al., ArXiv Astrophysics eprint:astro-ph/0803.0547 (2008).

[32] J. Lee, U.-L. Pen, Astrophys. J. , 555, 106 (2001).

[33] R.G. Crittenden, N. Priyamvada, U.-L. Pen, T. Theuns, Astrophys. J. , 559, 552 (2001).

[34] $P$ contains infinitely many $j=0$ (irreducible) representations of $\mathrm{SO}(3)$.

[35] Harish Chandra, Am. J. Phys. , 80, 241 (1958).

[36] C. Itzykson, J.B. Zuber J.B., J. Math. Phys. , 21, 411 (1980).

[37] R.J. Muirhead, Aspects of Multivariate Statistical Theory (Wiley, New-York, 1986)

[38] P. Koev and A. Edelman, Math. Comp. , 75, 833 (2006) for an algorithm linear in the size of the matrix arguments.

[39] J. Berntsen, T.O. Espelid, A. Genz, ACM Transactions on Mathematical Software, 17, 452.

[40] S.F. Shandarin, A.A. Klypin, Soviet Astron., 28, 491 (1984); C.S. Frenk, S.D.M. White, M. Davis, G. Efstathiou, Astrophys. J. , 327, 507 (1988); N. Katz, T. Quinn, J.M. Gelb, Mon. Not. R. Astron. Soc. , 265, 689 (1993); C. Porciani, A. Dekel, Y. Hoffman, Mon. Not. R. Astron. Soc., 332, 339 (2002).

[41] N. Kaiser, Astrophys. J., 284, 9 (1984). 
[42] U.L. Pen, J. Lee, U. Seljak, Astrophys. J. , 543, L107 (2001).

[43] J. Lee, U.-L. Pen, ArXiv Astrophysics eprint:astroph/0707.1690 (2007)

[44] C.M. Hirata, U. Seljak, Phys. Rev. D. , 70, 063526 (2004).

[45] R.E. Smith, P.I.R. Watts, Mon. Not. R. Astron. Soc., 360, 203 (2005); R.E. Smith, P.I.R. Watts, R.K. Sheth, Mon. Not. R. Astron. Soc. , 365, 214 (2006).

[46] S. Matarrese, L. Verde, R. Jimenez, Astrophys. J. , 541, 10 (2000).
[47] E. Komatsu, D.N. Spergel, Phys. Rev. D. , 63, 063002 (2001).

[48] J. Maldacena, Journal of High Energy Physics, 5, 13 (2003).

[49] L. Hui, Z. Zhang, ArXiv Astrophysics eprint:astro-ph/0205512.

[50] J.R. Bond, S.T. Myers, Astrophys. J. Suppl. , 103, 1 (1996).

[51] $\zeta_{11}$ is always less than $(13 \pi-27) / 30 \pi \approx 0.147$, a value reached only when $R_{1}=R_{2}$. 